\title{
Editorial
}

\section{Cyber-Physical Systems in Manufacturing and Service Systems}

\author{
Qing Chang, ${ }^{1}$ Robert Gao, ${ }^{2}$ Yong Lei, ${ }^{3}$ Lihui Wang, ${ }^{4}$ and Changxu Wu ${ }^{5}$ \\ ${ }^{1}$ Department of Mechanical Engineering, Stony Brook University, Stony Brook, NY 11794, USA \\ ${ }^{2}$ Department of Mechanical and Aerospace Engineering, Case Western Reserve University, Cleveland, OH 44106, USA \\ ${ }^{3}$ State Key Laboratory of Fluid Power Transmission and Control, School of Mechanical Engineering, Zhejiang University, \\ Hangzhou 310027, China \\ ${ }^{4}$ Department of Production Engineering, KTH Royal Institute of Technology, 10044 Stockholm, Sweden \\ ${ }^{5}$ Department of Industrial \& System Engineering, State University of New York at Buffalo, Buffalo, NY 14260, USA
}

Correspondence should be addressed to Qing Chang; qing.chang@stonybrook.edu

Received 17 September 2015; Accepted 8 October 2015

Copyright (C) 2015 Qing Chang et al. This is an open access article distributed under the Creative Commons Attribution License, which permits unrestricted use, distribution, and reproduction in any medium, provided the original work is properly cited.

Cyber-physical systems (CPS) are engineered systems that are built from and depend upon the seamless integration of computational algorithms and physical components. Unlike traditional embedded systems that are typically stand-alone, a full-fledged CPS is characterized by a network of interacting elements with physical input and output, resembling the structure of a sensor network. Tremendous progress has been made in advancing CPS technology over the last five years. New smart CPS will drive innovation and competition in sectors as diverse as aerospace, automotive, and chemical process, civil infrastructure, energy, healthcare, manufacturing, transportation, and so forth. One example of CPS is an intelligent manufacturing line, where the machine can perform a variety of processes by communicating with the components. Ongoing advancement in science and engineering will continue to enhance the link between computational and physical elements, dramatically increasing the adaptability, autonomy, efficiency, functionality, reliability, safety, and usability of cyber-physical systems.

This special issue is focused on the CPS in manufacturing and service systems. New CPS advancements in mathematical modeling, control, and optimization for complex engineering systems, especially applications in manufacturing, transportation, and energy, are emphasized. Topics covered in this issue include (i) CPS modeling, control and optimization for networked manufacturing processes, systems, and applications, (ii) intelligent sensing technology and instrumentations, and (iii) smart control and energy management in industries and intelligent transportation systems.
Seven papers have been included in the special issue, focusing on the subject "CPS modeling, control, and optimization for networked manufacturing processes, systems, and applications." The paper entitled "An Exact Method for the Analysis of a Two-Machine Manufacturing System with a Finite Buffer Subject to Time-Dependent Failure," by B. Xia et al., presents an efficient analytical method for the performance evaluation of a two-machine manufacturing system with a finite buffer, where the buffer is prone to time-dependent failure. The research result can be applied to accumulating conveyors, which are widely used in real industry systems. Y. Cao et al. in "Study on Resource Configuration on Cloud Manufacturing" consider the tolerance design with multiple candidate suppliers on cloud manufacturing platform to enable a two-level game optimization by considering the benefit of customers and the profit gain. In "Unit-Specific Event-Based and Slot-Based Hybrid Model Framework with Hierarchical Structure for Short-Term Scheduling," Y. Wang et al. study a production short-term scheduling problem through a two-layer structure of unit-specific event-based and slot-based hybrid model. The resource constraints and calculation accuracy are simultaneously considered. In "Application of Two-Phase Fuzzy Optimization Approach to Multiproduct Multistage Integrated Production Planning with Linguistic Preference under Uncertainty," S. Lu et al. propose a fuzzy multiobjective mixed integer programming model with different objective priorities to simultaneously minimize the relevant operation cost and maximize the average safety stock holding level 
and service level. J. Xin et al. in "An Improved Generalized Predictive Control in a Robust Dynamic Partial Least Square Framework" propose a new robust dynamic partial least squares (PLS) model based on an outliers detection method using an improved radial basis function and a hidden Markov model (HMM). In "Error Modeling and Compensation of Circular Motion on a New Circumferential Drilling System," Q. Fang et al. propose a new flexible circumferential drilling system to be used on the fuselage docking area, where the influence of the circular motion error to the drilling accuracy is studied and a laser tracker is utilized to measure the circular motion error. For the analysis of the mixed new and remanufactured product market, B. Wang and J. Wang in "Price and Service Competition between New and Remanufactured Products" investigate the optimal decisions of prices, service levels, demands, and profits in three scenarios: Manufacturers Stackelberg, Retailer Stackelberg, and Nash Equilibrium.

There are three contributions that are closely related to intelligent sensing technology and instrumentations. In "A Topology Evolution Model Based on Revised PageRank Algorithm and Node Importance for Wireless Sensor Networks," X. Qi et al. describe the design of the preferential attachment method through a revised PageRank algorithm and the dynamics of the network topology evolution through theoretical and simulation analysis. Q. Fang et al. in "A Force Sensorless Method for CFRP/Ti Stack Interface Detection during Robotic Orbital Drilling Operations" propose a force sensorless method based on cutting force observer, which combined an adaptive disturbance observer and friction force model, to monitor the thrust force and identify the drilling material during the drilling process of carbon fiber reinforced plastics and titanium (CFRP/Ti) stacks. In "Modified Extended Kalman Filtering for Tracking with Insufficient and Intermittent Observations," P. Chen et al. investigate the tracking of a single target on the fixed-topology wireless sensor networks (WSNs) using a modified extended Kalman filtering algorithm.

There are two papers that are concerned with smart control and energy management in industries and intelligent transportation systems. K. E. M. Reena et al. in "An Occupancy Based Cyber-Physical System Design for Intelligent Building Automation" present a novel architecture of CPS for wireless networked intelligent building automation system (IBAS) with priority-based access mechanism for zones in a large building with dynamically varying occupancy, and an algorithm is proposed for power scheduling in sensor nodes to reduce their energy consumption. Priority status of zones based on occupancy is determined using fuzzy inference engine, and Nondominated Sorting Genetic Algorithm-II is used to solve the optimization problem involving conflicting demands of minimizing total energy consumption and maximizing occupant comfort levels in buildings. In "Addressing the Safety of Transportation Cyber-Physical Systems: Development and Validation of a Verbal Warning Utility Scale for Intelligent Transportation Systems," Y. Zhang et al. investigate driving safety improvement by informing drivers of hazards with warning in advance. The effectiveness of a warning in preventing accidents is evaluated and a driving simulator study is conducted to examine the Verbal Warning Utility Scale (VWUS) in a simulated driving environment.

\section{Acknowledgments}

This special issue represents an exciting and focused snapshot of current research on CPS in manufacturing and service systems. We would like to take this opportunity to thank all the contributing authors who have submitted their papers for review in the special issue. In particular, we express our sincere gratitude to all the reviewers who devoted their time to review the submitted manuscripts. It is their selfless service to the community that has helped made this special issue a reality. 


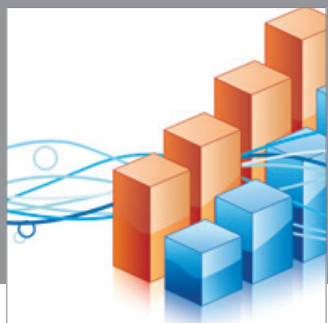

Advances in

Operations Research

mansans

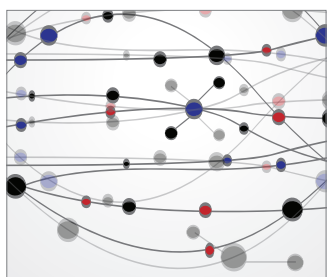

The Scientific World Journal
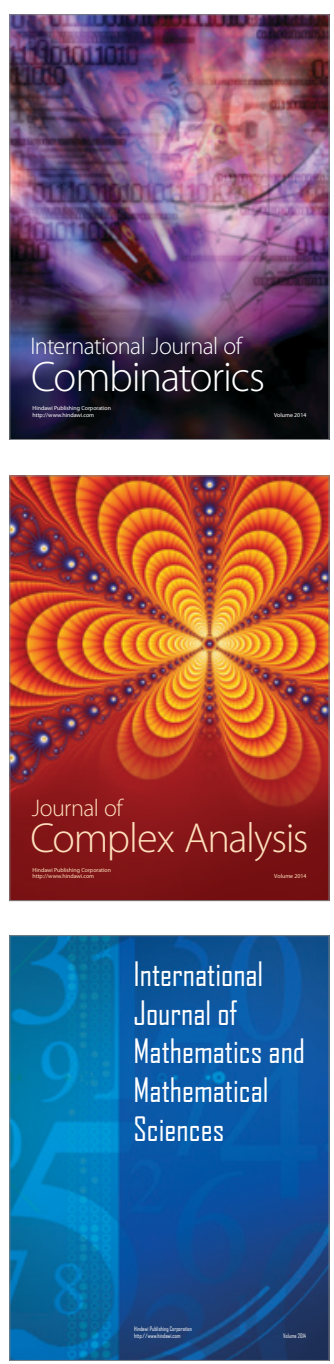
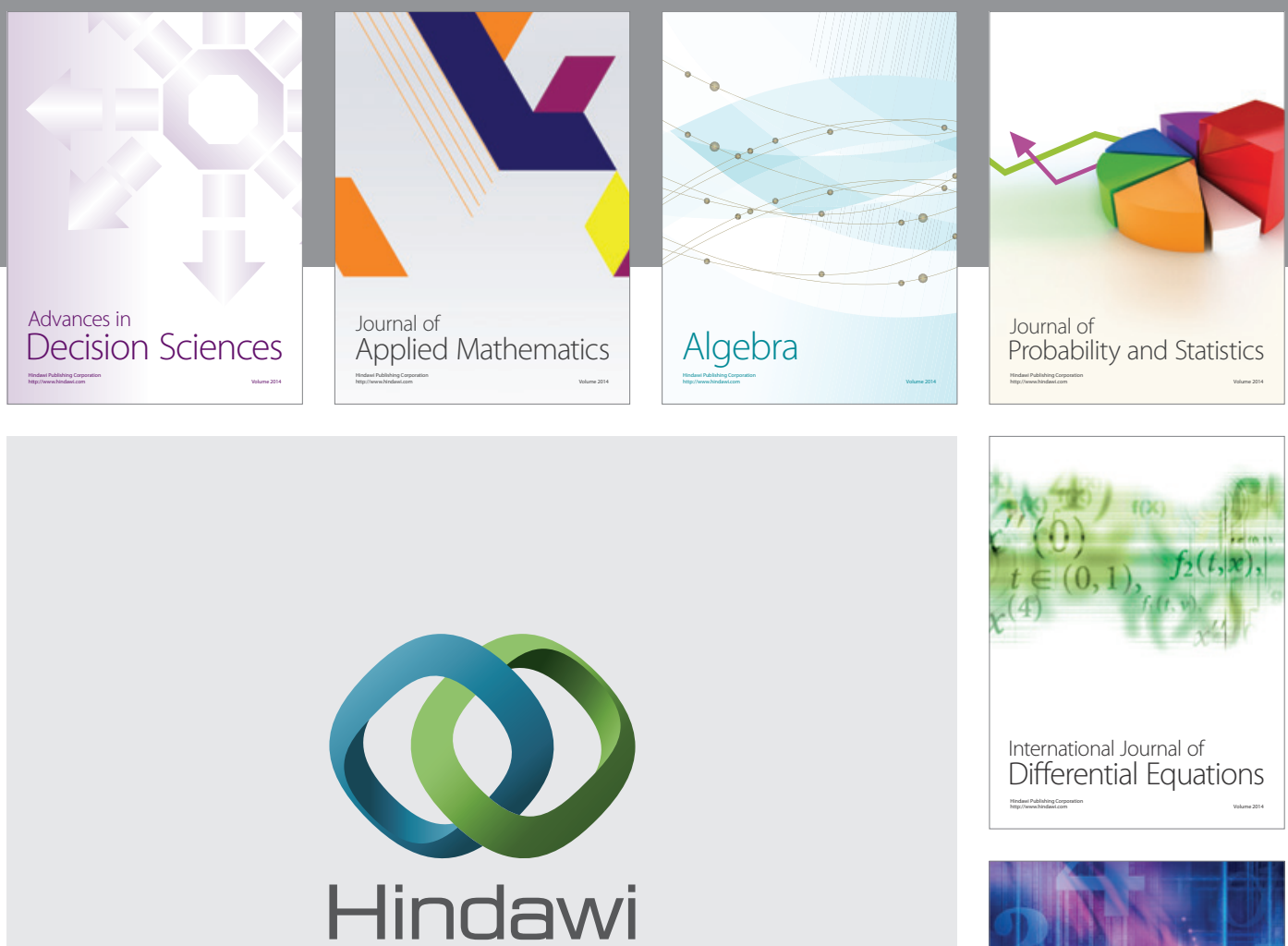

Submit your manuscripts at http://www.hindawi.com
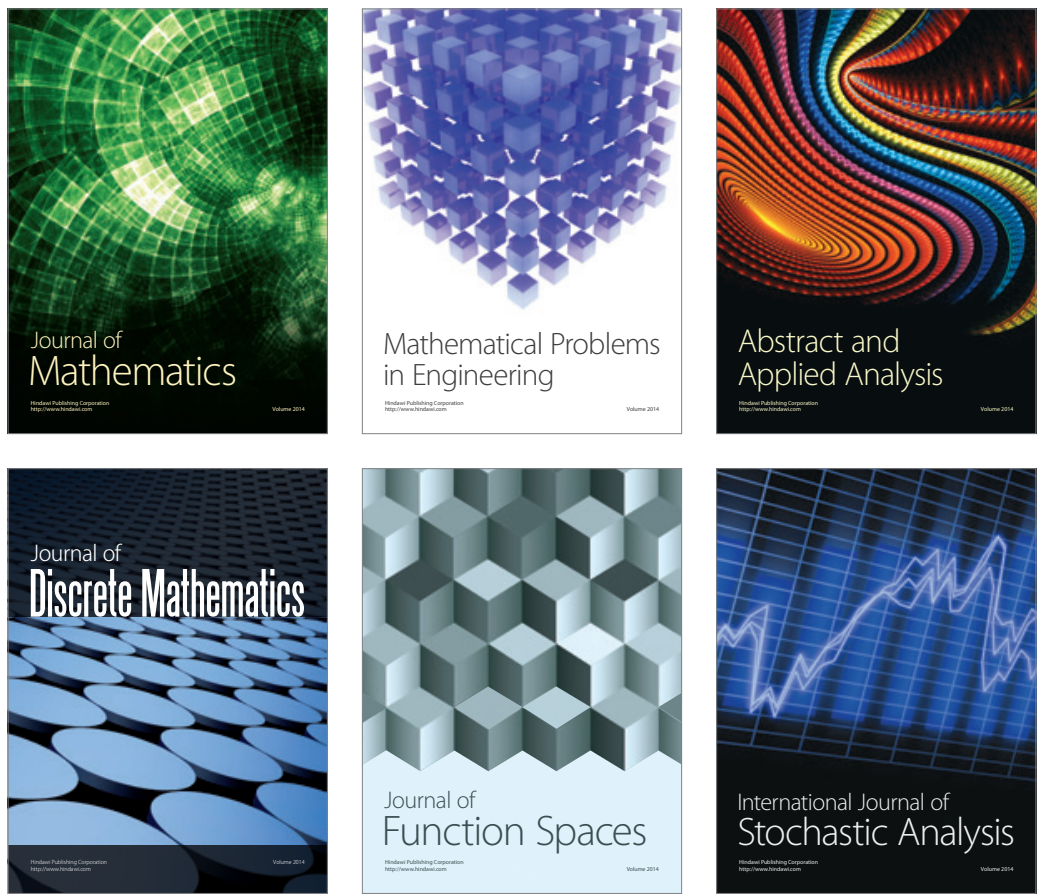

Journal of

Function Spaces

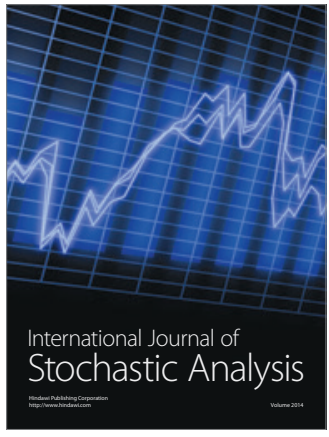

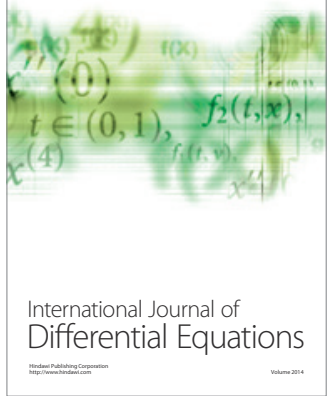
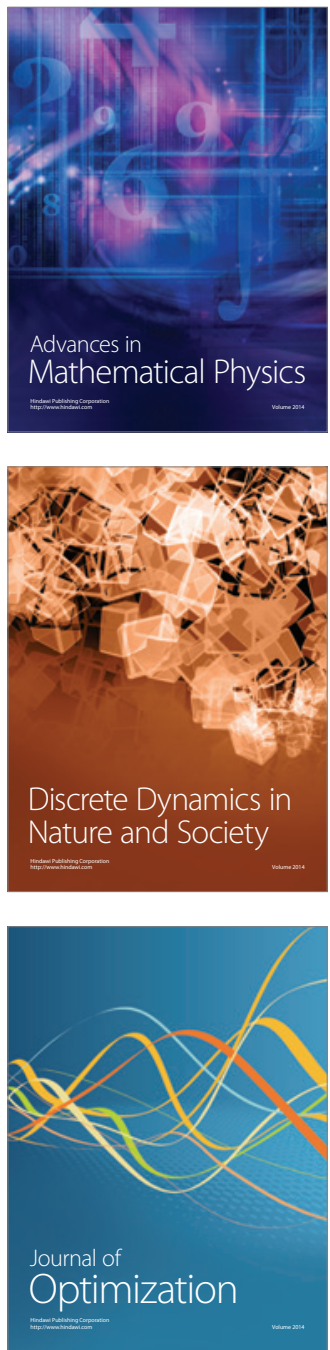